
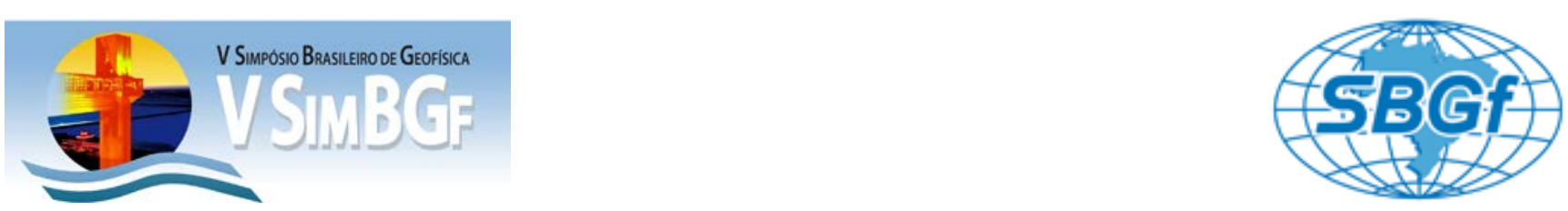

\title{
Investigação rasa com o método de análise multicanal das ondas superficiais empregando conjuntamente fontes passiva e ativa
}

\author{
Júlio Cesar Ardito, Renato Prado, Universidade de São Paulo
}

Copyright 2012, SBGf - Sociedade Brasileira de Geofísica

Este texto foi preparado para a apresentação no V Simpósio Brasileiro de Geofísica, Salvador, 27 a 29 de novembro de 2012. Seu conteúdo foi revisado pelo Comitê Técnico do $V$ SimBGf, mas não necessariamente representa a opinião da SBGf ou de seus associados. É proibida a reprodução total ou parcial deste material para propósitos comerciais sem prévia autorização da SBGf.

\section{Resumo}

O presente estudo de caso consistiu na aplicação do método da analise multicanal de ondas superficiais em uma área cuja litologia é bem conhecida. Neste trabalho foram usadas fontes ativas em conjunto com passivas (Roadside). Os resultados mostraram um modelo de velocidades de onda $S$ dos primeiros $60 \mathrm{~m}$ abaixo da superfície. Essas velocidades foram correlacionadas com dados de poço e o método mostrou precisão significativa no mapeamento da interface solo/rocha.

\section{Introdução}

A busca por técnicas sísmicas de exploração rasa que sejam aplicáveis em áreas urbanas onde o ruído é elevado e inviabiliza a aplicação das técnicas tradicionais, motivou o emprego de fontes passivas em conjunto com o método da análise multicanal de ondas superficiais, conhecido como MASW (multi-channel analysis of surfase waves). O método permite mapear as variações horizontais e verticais do campo de velocidades da onda S e, eventualmente, se chegar aos módulos de cisalhamento, se bem conhecidas às densidades dos materiais investigados (Xia et al, 1997; Xia et al, 1998; Park et al, 1999). Além disso, nas áreas urbanas, a análise das ondas sísmicas de longo comprimento geradas por fontes passivas permite mapear profundidades maiores do que as usualmente obtidas com o uso de fontes ativas, ou com o emprego do método sísmico de refração, neste último caso em razão das limitações impostas pelo meio.

O método tem se mostrado eficiente para diversas finalidades como, por exemplo, para o mapeamento litológico abaixo de pavimentos de concreto (Ryden et al., 2003; Park et al., 2001), na caracterização de solos (Ivanov et al., 2000; Park \& Miller, 2005a; 2005b; Xia et al.,1999; 2000) e na determinação do topo rochoso e identificação de fraturas (Miller et al.,1999b; Miller et al.,2000).

Inicialmente o MASW foi desenvolvido empregando-se apenas fontes ativas. Posteriormente, Park e Miller (2006) desenvolveram uma técnica mais funcional para registrar ondas superficiais provenientes de fontes passivas em áreas urbanas ou ao longo de estradas. Esta técnica foi denominada Passive Road Side MASW e consiste no emprego de um arranjo linear de geofones dispostos paralelamente a uma rua movimentada ou a uma estrada, de modo a utilizar as baixas frequências emitidas pelo tráfego de veículos.

Embora já bem consolidado na América do Norte e Europa, o estudo e aplicação do método ainda é recente no Brasil, mesmo no âmbito acadêmico, existindo apenas uma tese de mestrado (Lima Júnior, 2007) sobre o tema, porém em nenhum destes as fontes passivas foram empregadas.

O principal objetivo do presente estudo de caso é aplicar o método da análise multicanal de ondas superficiais utilizando fontes passivas e ativas em conjunto, de modo a alcançar uma profundidade maior (cerca de 60m) sem perder resolução nas camadas mais rasas (até $30 \mathrm{~m}$ de profundidade). Para essa finalidade foi escolhida uma área de geologia bem conhecida - sítio controlado do IAG-USP (Porsani et al.,2004) - localizada em ambiente urbano.

\section{Metodologia/ Problema Investigado}

O presente estudo foi realizado no sítio controlado do IAG/USP por ser um local cuja subsuperfície já foi bem investigada através de diferentes métodos geofísicos, e também por meio de investigações diretas com a execução de três sondagens mistas (percussão e rotativa) com amostragem (figura 1 ).

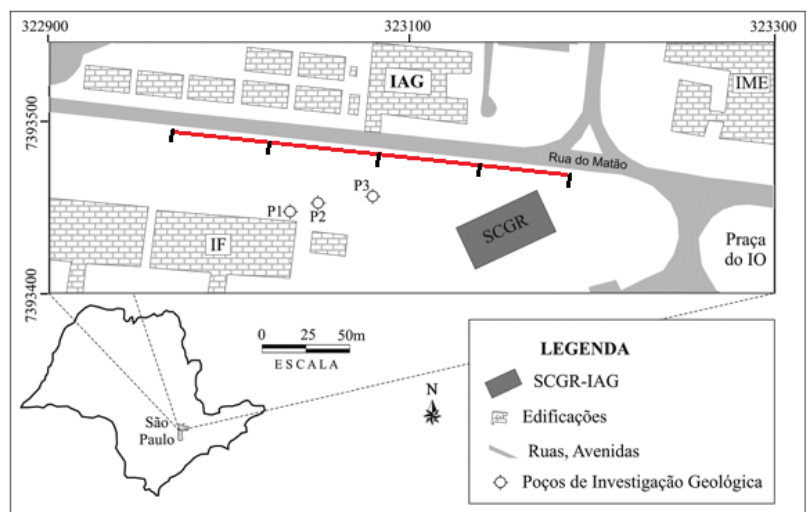

Figura 1: Croqui do sítio controlado do IAG/USP com identificação do posicionamento da linha sísmica executada e a localização dos furos de sondagem de referência (Porsani et al., 2004).

Como pode ser observado na Figura 1, foi empregado um arranjo linear de geofones, com centro coincidente com a localização da sondagem P3. 
A técnica adotada para o emprego de fonte passiva foi a Passive Roadside (Park e Miller, 2006), aproveitando o ruído proveniente do tráfego de veículos da Rua do Matão, principalmente ao passarem por duas lombadas ali existentes (Figura 1 e Figura 2). Além disso, foi usada uma marreta de $8 \mathrm{~kg}$ como fonte ativa em uma aquisição complementar. O comprimento total do arranjo foi o mesmo para ambos os ensaios, porém na aquisição com fonte ativa utilizou-se 96 geofones, e na passiva 48, adotando-se espaçamentos entre geofones de $1,5 \mathrm{~m}$ e 3 $\mathrm{m}$, respectivamente. Foram empregados geofones de 4,5 $\mathrm{Hz}$ de frequência natural.

As fontes ativas usualmente geram ondas de mais alta frequência, que podem mapear os horizontes mais superficiais, já as fontes passivas geram ondas de menor frequência e por isso podem imagear até cerca de $100 \mathrm{~m}$ de profundidade (Park et al., 2005). A aquisição ativa forneceu uma curva de dispersão com intervalo de mais alta frequência (20-40 Hz), e a aquisição passiva curvas de dispersão com frequências mais baixas (5-15 Hz).

Para o processamento dos dados, extração da curva de dispersão e a inversão utilizou-se o software Surfseis 2.0 desenvolvido pelo Kansas Geological Survey.

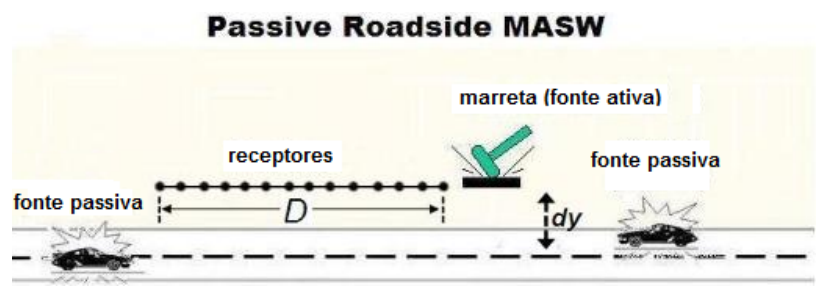

Figura 2: Modelo esquemático de aquisição pela técnica Passive Roadside (Fonte: www.masw.com).

\section{Resultados}

No processamento dos dados foi utilizado o software SurfSeis 2.0. Nesta etapa os dados foram divididos em dois conjuntos, ativos e passivos. Uma análise criteriosa das curvas de dispersão mostrou que as geradas a partir dos dados obtidos com fonte passiva apresentaram um pequeno deslocamento em relação às curvas dos dados de fonte ativa. Uma hipótese que explica esta variação esta embasada na classe da frente de onda registrada. Segundo Park et. al. (2006) quando se tem frentes de ondas planas e que são registradas na mesma linha em que se encontra o arranjo de geofones, são produzidas curvas de dispersão com uma ligeira diferença em relação às ondas planas registradas fora da linha do arranjo de sensores.

Os modelos produzidos a partir da fonte ativa mostraram velocidades variando entre 600 e $800 \mathrm{~m} / \mathrm{s}$ e profundidades entre 0 e $24 \mathrm{~m}$ (Figura 4).
Após a profundidade de $24 \mathrm{~m}$ houve grande variação entre modelos de fonte ativa, indicando ser esta a profundidade limite de investigação empregando-se a fonte ativa, na situação geológica investigada.

No caso do conjunto de dados gerados com o emprego da fonte passiva (Figura 3), observou-se que as curvas de dispersão tiveram o modo fundamental concentrado em uma faixa de frequência que variou entre 4 e $15 \mathrm{~Hz}$. O modelo final foi correlacionado com a litologia do poço (Figura 5) e as interfaces apresentaram variações de cerca de 15\% em relação ao perfil.

\section{Discussão e Conclusões}

A aquisição ativa com a marreta permitiu imagear os primeiros $25 \mathrm{~m}$ abaixo da superfície e mostrou espessuras compatíveis com a litologia.

O modelo produzido a partir dos dados obtidos com a fonte passiva foi capaz de imagear profundidades de até $60 \mathrm{~m}$. As interfaces das camadas apresentaram compatibilidade significativa (cerca de 15\%) entre 0 modelo e as informações do perfil da sondagem, principalmente o contato entre o embasamento e a argila compactada. Os modelos ativo e passivo não mostraram compatibilidade de velocidades, embora as interfaces sejam compatíveis.

O uso combinado de fontes ativas e passivas permitiu ampliar a faixa de frequência, melhorando a curva de dispersão. Desta forma, foi possível obter melhor resolução nas camadas mais rasas e ainda atingir profundidades da ordem de $60 \mathrm{~m}$.

\section{Referências}

Lima Junior, S. B., 2008. Emprego das ondas Rayleigh visando à caracterização geotécnica de solos superficiais de encosta numa área teste em Ubatuba/SP. Rev. Bras. Geof. , 26 (2), 239-239.

Miller, R. D., and Xia, J., 1999a, Feasybility of seismic techniques to delineate dissolution features in the upper $600 \mathrm{ft}$ at Alabama Eletric Coperative's proposed Damascus site: Kansas Geological Survey Open-File Report 99-3.

Miller, R.D., Xia, J., Park, C.B., and Ivanov, J.M., 1999, Multichannel analysis of surface waves to map bedrock, Kansas Geological Survey, The Leading Edge, December, p. 1392-1396.

Park, C.B., Miller, R.D., and Xia, J., 1996, Multi-channel analysis of surface waves using Vibroseis (MASWV): [Expanded Abstract]: Soc. Explor. Geophys., 68-71. 
Park, C.B., Miller, R.D., and Xia, J., 1999, Multi-channel analysis of surface waves (MASW): Geophysics, v. 64, no. 3, p. 800-808.

Park, C.B., R.D. Miller, J. Xia, J. Ivanov, J.A. Hunter, R.L. Good, and R.A. Burns, 2000a, Multichannel analysis of underwater surface waves near Vancouver, B.C., Canada [Exp. Abs.]: Soc. Expl. Geophys., p. 1303-1306.

Park, C.B., Ivanov, J., Miller, R.D., Xia, J., and Ryden, N., 2001a, Multichannel analysis of surface waves (MASW) for pavement-feasibility test: Proceedings of the 5th SEGJ International Symposium, Tokyo, p. 25-30.

Park, C.B.; Miller, R.D.; Ryden N.; Xia, J.; Ivanov, J., 2005 - Combined use of active and passive surface waves. Journal of Engineering and Environmental Geophysics, v.10 (3), pg. 323-334.

Park, C.B.; Miller, R.D., 2006 - Roadside passive MASW. Proceedings of the 19th Symposium on the Application of Geophysics to Engineering and Environmental Problemas - SAGEEP. Society of Engineering and Environmental Geophysics. Seattle, Washington. $12 \mathrm{p}$.
Xia, J., Miller, R.D., and Park, C.B., 1997, Estimation of shear wave velocity in a compressible Gibson half-space by inverting Rayleigh wave phase velocity: Technical Program with Biographies, SEG, 67th Annual Meeting, Dallas, TX, 1917-1920.

Xia, J., Miller R.D., and Park, C.B., 1998, Construction of vertical section of near-surface shear-wave velocity from ground roll: Technical Program, The Society of Exploration Geophysicists and The Chinese Petroleum Society Beijing 98' International Conference, 29-33.

Xia, J., Miller, R.D., and Park, C.B., 1999, "Estimation of near-surface shear-wave velocity by inversion of Rayleigh wave," Geophysics, 64(3), 691-700.

Xia, J., R.D. Miller, C.B. Park, and J. Ivanov, 2000, Construction of 2-D vertical shear-wave velocity field by the multichannel analysis of surface wave technique: Proceedings of the Symposium on the Application of Geophysics to Engineering and Environmental Problems (SAGEEP 2000), Arlington, Va., February 20-24, p. 11971206.

PORSANI, J.L.; BORGES, W.R.; ELIS, V.R.; DIOGO, L.A.; HIODO, F.Y.; MARRANO, A. \& BIRELLI, C.A., 2004. Investigações geofísicas de superfície e de poço no sítio controlado de geofísica rasa do IAG-USP.

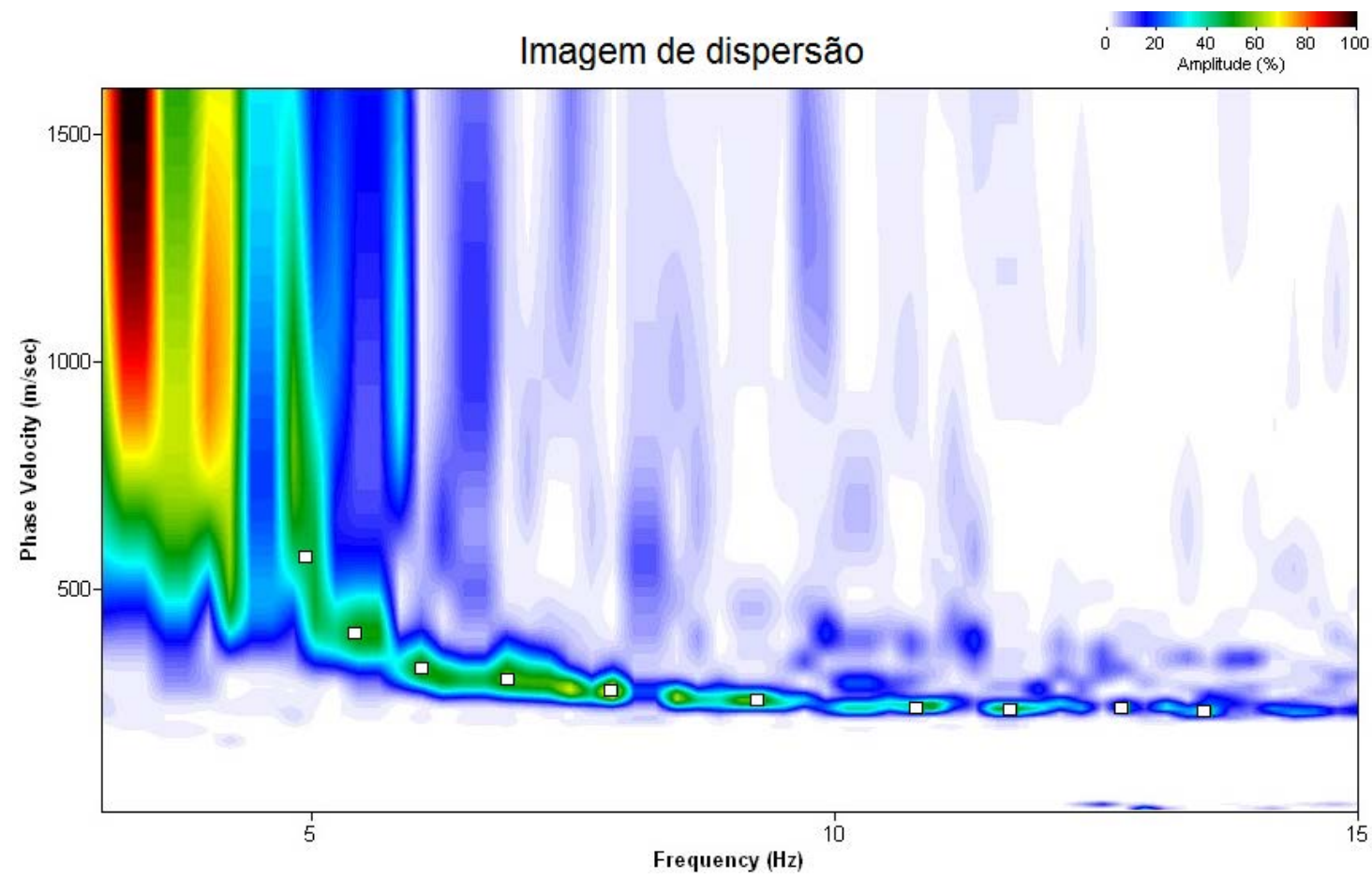

Figura 3: Diagrama da velocidade de fase pela frequência mostrando uma imagem de dispersão obtida a partir de fontes passivas. 


\section{Imagem de dispersão}

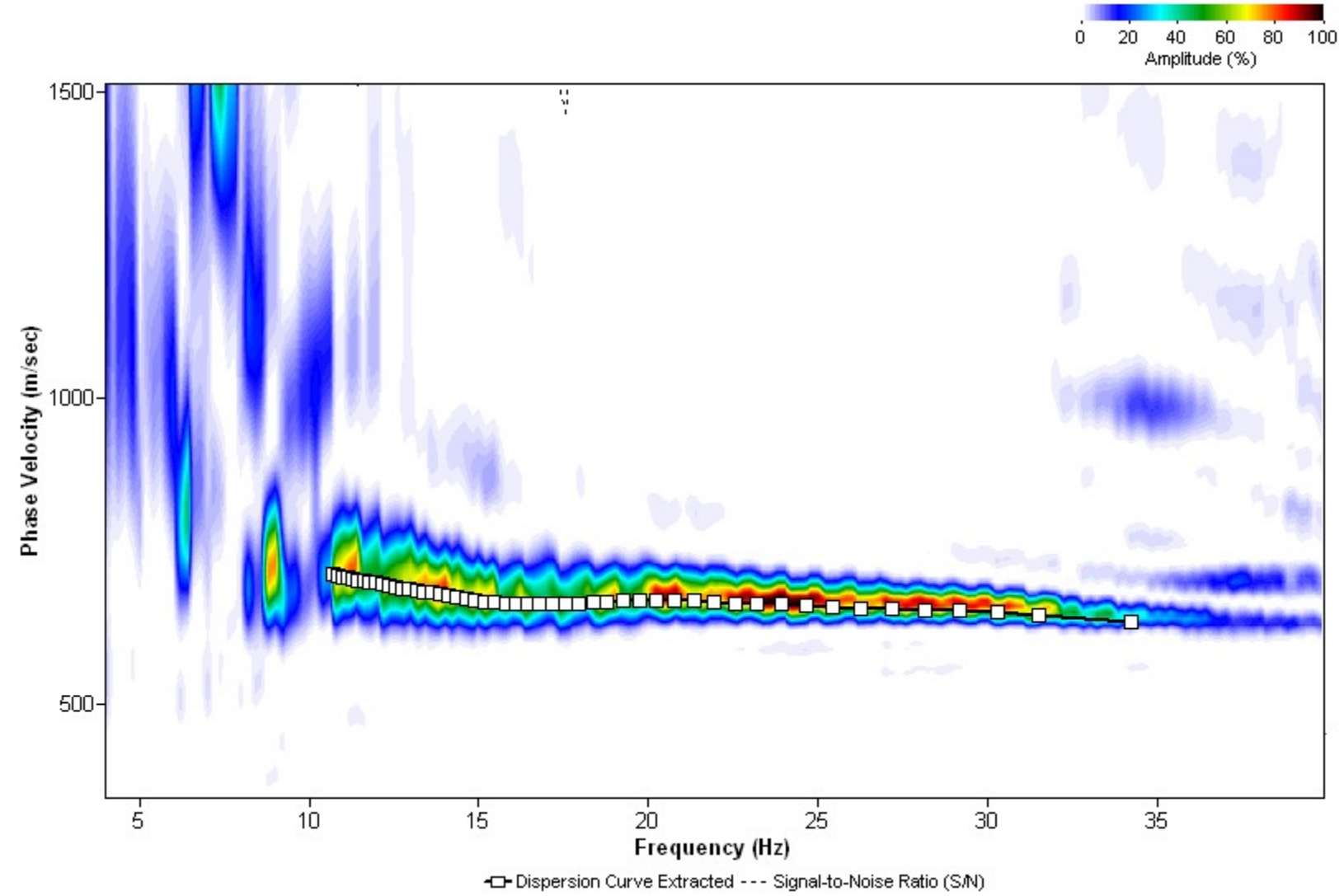

Figura 4: Diagrama da velocidade de fase pela frequência mostrando uma imagem de dispersão obtida a partir da fonte ativa (marreta). 


\section{Modelo de Velocidade da onda S}

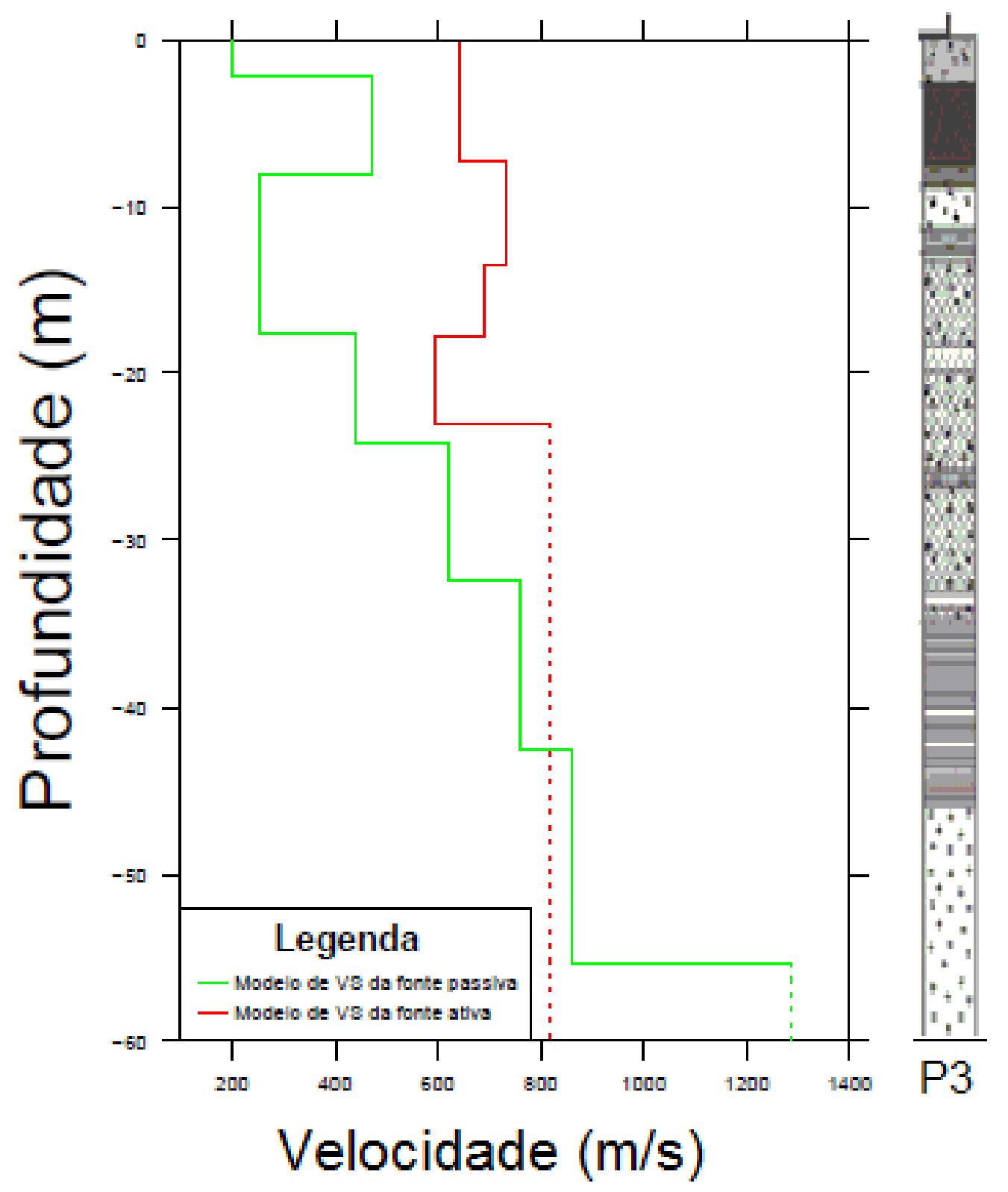

F:a Aterro areno-argiloso

Areia fina siltosa

Argila Siltosa
Argila siltosa com presença de matéria orgânica

88. Areia média a grossa, siltosa, com pedregulhos finos

$\therefore$ Granito Gnaisse

E-7. Argila siltosa

Figura 5: Correlação entre o modelo experimental e as informações da litologia do local estudado. 